\title{
Progress in controlling ICRF-edge interactions in ASDEX upgrade
}

VI. Bobkov', Ph. Jacquet, R. Ochoukov, W. Zhang, R. Bilato, F. Braun, D. Carralero, L. Colas, A. Czarnecka, R. Dux, H. Faugel, H. Fünfgelder, J. Jacquot, A. Křivská, T. Lunt, D. Milanesio, R. Maggiora, O. Meyer, I. Monakhov, J.-M. Noterdaeme, S. Potzel, Th. Pütterich, I. Stepanov, and the ASDEX Upgrade Team

Citation: AIP Conference Proceedings 1689, 030004 (2015); doi: 10.1063/1.4936469

View online: http://dx.doi.org/10.1063/1.4936469

View Table of Contents: http://aip.scitation.org/toc/apc/1689/1

Published by the American Institute of Physics 


\title{
Progress in Controlling ICRF-edge Interactions in ASDEX Upgrade
}

\author{
Vl. Bobkov $^{1, a)}$, Ph. Jacquet ${ }^{4)}$, R. Ochoukov ${ }^{1)}$, W. Zhang ${ }^{1,7)}$, R. Bilato ${ }^{1)}$, F. Braun ${ }^{1)}$, \\ D. Carralero ${ }^{1)}$, L. Colas ${ }^{2)}$, A. Czarnecka ${ }^{3)}$, R. Dux ${ }^{1)}$, H. Faugel ${ }^{1)}$, H. Fünfgelder ${ }^{1)}$, \\ J. Jacquot ${ }^{1)}$, A. Křivskás), T. Lunt ${ }^{1)}$, D. Milanesio ${ }^{6)}$, R. Maggiora ${ }^{6)}$, O. Meyer ${ }^{2}$, \\ I. Monakhov ${ }^{4)}$, J.-M. Noterdaeme ${ }^{1,7)}$, S. Potzel ${ }^{1)}$, Th. Pütterich ${ }^{1)}$, I. Stepanov ${ }^{1,7)}$ \\ and the ASDEX Upgrade Team
}

\author{
${ }^{1}$ Max-Planck-Institut für Plasmaphysik, Boltzmannstr.2, D-85748, Germany \\ ${ }^{2}$ CEA, IRFM, F-13108 Saint-Paul-lez-Durance, France \\ ${ }^{3}$ Institute of Plasma Physics and Laser Microfusion, Hery 23 Str., 01-497 Warsaw, Poland \\ ${ }^{4}$ CCFE, Culham Science Centre, Abingdon, OX14 3DB, UK \\ ${ }^{5}$ Laboratory for Plasma Physics, ERM/KMS, EUROfusion Consortium Member \\ ${ }^{6}$ Department of Electronics, Politecnico di Torino, Torino, Italy \\ ${ }^{7}$ Department of Applied Physics, Gent University, 9000 Gent, Belgium \\ ${ }^{a)}$ Corresponding author: bobkov@ipp.mpg.de
}

\begin{abstract}
RF measurements during variation of the strap voltage balance of the original 2-strap ICRF antenna in ASDEX Upgrade at constant power are consistent with electromagnetic calculations by HFSS and TOPICA, more so for the latter. RF image current compensation is observed at the antenna limiters in the experiment at a local strap voltage of about half of the value of the remote strap, albeit with a non-negligible uncertainty in phasing. The RF-specific tungsten (W) source at the broad-limiter 2-strap antenna correlates strongly with the RF voltage at the local strap at the locations not connected to opposite side of the antenna along magnetic field lines. The trends of the observed increase of the RF loading with injection of local gas are well described by a combined EMC3-Eirene - FELICE calculations, with the most efficient improvement confirmed for the outer-midplane valves, but underestimated by about $1 / 3$. The corresponding deuterium density tailoring is also likely responsible for the decrease of local $\mathrm{W}$ sources observed in the experiment.
\end{abstract}

\section{INTRODUCTION AND EXPERIMENTAL SETUP}

Development of Ion Cyclotron Range of Frequencies (ICRF) systems for reactor-relevant magnetic fusion devices requires implementation of strategies to reduce the accompanying parasitic RF sheath effects on one hand and to increase the RF power capability on the other.

Several strategies of antenna design development aimed to reduce the RF sheath effects have been proposed and partially implemented recently [1-3]. Assessment of the role of antenna design in experiments is generally not straightforward. In this paper, we discuss the near-antenna measurements as well as the measurements on the field lines connected to an antenna (parallel ion energy) in combination with the variations of antenna strap voltage balance and strap phasing as tools to evaluate antenna designs experimentally in ASDEX Upgrade (AUG). The strap voltage balance and the phasing flexibility has been made possible by the recent upgrade of the ICRF system in preparation of the new AUG three-strap antenna installation in 2015 [2].

The gas injection technique to increase the local plasma density and therefore the RF loading $[4,5]$ is reliably used in AUG and in JET [6]. Furthermore it is beneficial for reduction of impurity production. The intrinsically 3D plasma density distribution in front of the antennas is now simulated by the plasma transport code EMC3-Eirene [7] that solves Braginskii fluid equations and is self-consistently coupled to the kinetic neutral transport code Eirene. 
The actuator flexibility in AUG allows to control the ICRF-edge interactions, if not always to minimize them, and achieve a better understanding of the RF sheath and the density tailoring effects, before the newer antenna and gas valve designs are implemented.

The standard ICRF heating scheme of hydrogen minority in deuterium in $\mathrm{H}$-mode discharges is used in the AUG experiments discussed below. Figure 1 shows the positions of four ICRF antennas, $a 1$ to $a 4$, of gas valves and of the relevant local diagnostics including limiter spectroscopy at a4 (broad-limiter antenna) measuring WI emission and the limiter RF shunt measurements at a2 (original AUG antenna). The $j \times B$-driven reciprocating retarding field analyzer (RFA) connects to the lower half of $a 4$ along magnetic field lines and

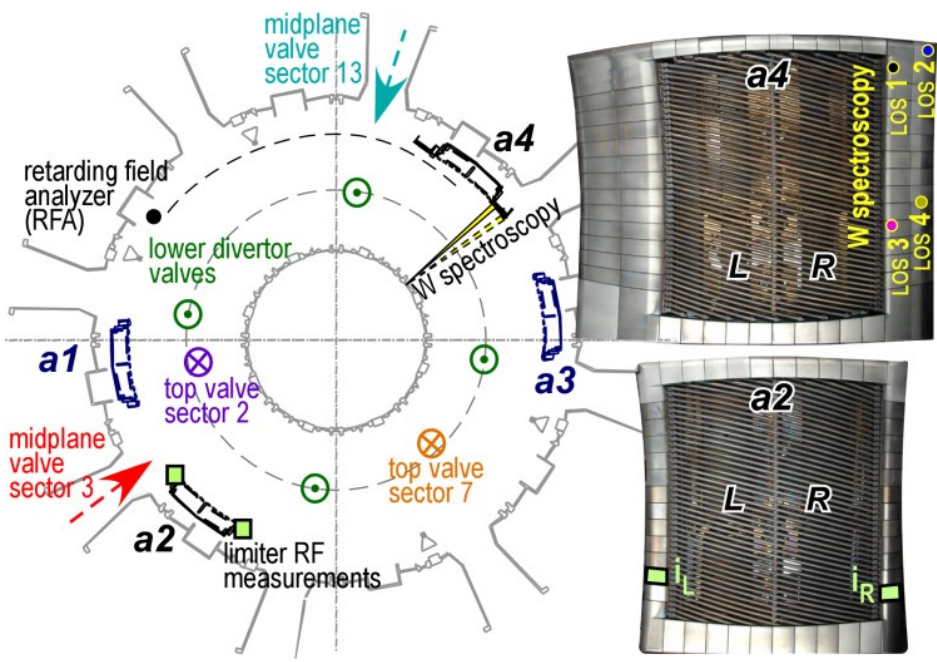

FIGURE 1. AUG toroidal view of ICRF antennas, gas valves and special diagnostics. measures averaged ion energies parallel to the magnetic field lines. As shown on the right side of the figure, antenna straps are distinguished as $L$ (left) and $R$ (right). The antennas are operated in pairs $a 1 / a 3$ and $a 2 / a 4$ within the $3 \mathrm{~dB}$ hybrid scheme and are matched by double-stub tuner systems. Antennas a2/a4 are equipped with individual strap feeders with strap phasing control (feed-back) and power control (no feedback). One RF generator feeds the $L$-straps of $a 2$ and $a 4$ and another feeds the $R$-straps, the phasing is feedback-controlled at $a 2$ based on the measurements of the RF forward power in the resonant lines. The changes of the strap feeder parameters at $a 2$ and $a 4$ are about synchronous. A de-synchronization in phasing between $a 2$ and $a 4$ can exist due to differences in RF matching and in electric line lengths of the feeders.

The gas system consists of the valves in the lower divertor, at midplane and at the top of the machine. We consider only 4 spectroscopic lines of sight (LOS) indexed from 1 to 4 , for the $a 4 \mathrm{~W}$ spectroscopy. The trends in the observations of the $\mathrm{W}$ influx described in this paper can be directly applied to the trends of the $\mathrm{W}$ sputtering yield. The $a 2$ limiter RF shunt measurements $i_{\mathrm{R}}$ and $i_{\mathrm{L}}$ monitor amplitudes of the local RF currents. Implementation of the electromagnetic (EM) simulations with HFSS (lossy dielectric loading) [9] and TOPICA (plasma loading with actual density and temperature profiles) [10] with a vacuum layer in front of the limiters [2] allows to treat $i_{\mathrm{R}}$ and $i_{\mathrm{L}}$ as the values proportional to the local absolute values of the electric field in front of the limiter. The parallel component of this electric field, being mainly a function of the local RF currents and the limiter geometry in the calculations mentioned above, is thought to be driving the RF sheath effects.

\section{VOLTAGE BALANCE CHARACTERISTICS AT DIPOLE PHASING}

In combination with the measurements of $i_{\mathrm{L}}$ and $i_{\mathrm{R}}$, the individually controlled antenna feeders at $a 2$ and $a 4$ provide an experimental validation of the EM calculations with HFSS and TOPICA, independently of the advanced RF sheath modeling.

Figure 2 compares $i_{\mathrm{L}}$ and $i_{\mathrm{R}}$ with the expected local amplitude of the RF electric field at the limiter from the EM calculations $\left|E_{\mathrm{RF}}\right|$ as a function of the ratio of the local strap RF voltage to the remote strap RF voltage at the antenna feeders, i.e. $V_{\mathrm{RF}} / V_{\mathrm{RF}}$ for $i_{\mathrm{L}}$ (left side of Fig. 2) and $V_{\mathrm{RF}} / V_{\mathrm{RF}} \mathrm{L}$ for $i_{\mathrm{R}}$ (right side of Fig. 2), for dipole strap phasing. The experimental conditions were optimized: high density (high deuterium gas
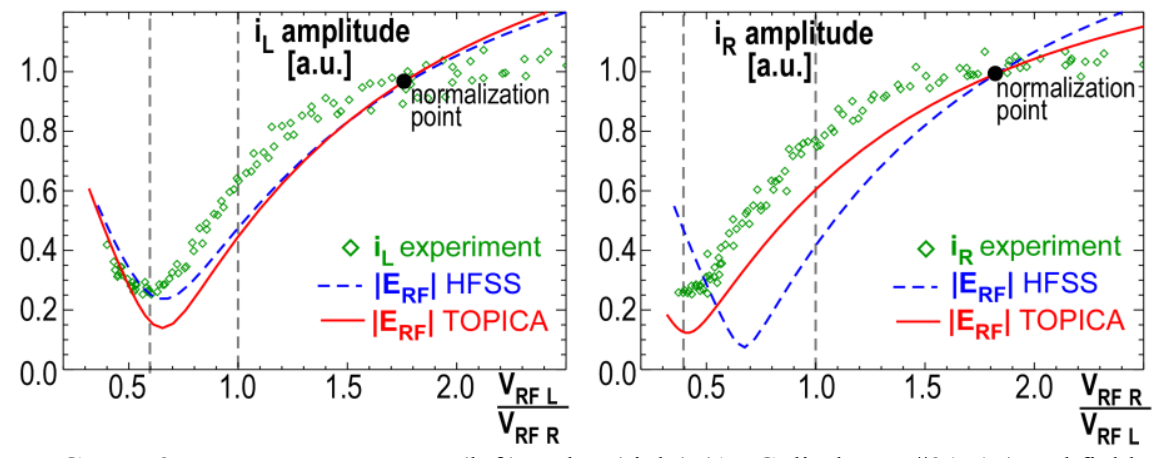

FIGURE 2. RF measurements $i_{\mathrm{L}}$ (left) and $i_{\mathrm{R}}$ (right) (AUG discharge \#31515) and field amplitudes $\left|E_{\mathrm{RF}}\right|$ from EM modeling of antenna a2 vs. strap voltage balance at dipole phasing. Vertical dashed lines: starndard ratio of 1.0 and ratios for minima in experiment. 
rate) $\mathrm{H}$-mode scenario at $B_{\mathrm{t}}=2.5 \mathrm{~T}$ was used with magnetic perturbation coils with only $1 \mathrm{MW}$ of total ICRF power at $36.5 \mathrm{MHz}$ on top of $5 \mathrm{MW}$ of NBI heating. The coils allowed to suppress ELMs and provide quiet ELM-free conditions in the SOL, and the low $\mathrm{P}_{\mathrm{ICRF}}$ allowed to keep to a minimum the non-linearities that are connected to possible changes of the ICRF heating in the core plasma during the strap voltage balance variation. Both in the experiment and in the calculations the launched ICRF power was kept constant throughout the voltage ratio scan. The vertical dashed lines show the standard strap voltage ratio of 1.0 and the voltage ratio for the minimum of the amplitude in the experiment. The calculations for the planar AUG antenna models were used

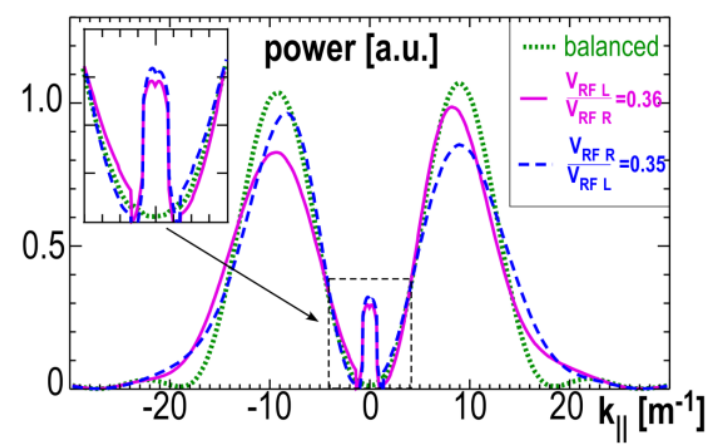

FIGURE 3. Poloidally integrated antenna $\mathrm{k}_{\|}$power spectrum depending on strap voltage balance. for the EM analysis, with the absolute values from the experiment and the calculations normalized at the ratio of voltages of 1.75 for the best fit.

Both the experimental and the calculated amplitude values show a minimum in the vicinity of the strap voltage ratio of 0.5 . This is consistent with expectation that at the minimum, the $(0, \pi)$ phased contributions of the image currents of the straps at the limiters cancel each other to a large extent. At even lower voltage ratio the RF image current of the remote strap becomes larger than that of the local strap and the local RF amplitude increases again. The voltage balance characteristics in Fig. 2 are encouragingly similar in the experiment and in the calculations. For the experimental conditions considered, the measured RF amplitudes at the limiters agree at least qualitatively to those expected from the EM calculations. However the agreement is not perfect and several factors can contribute.

On the calculations side, a refinement of the antenna models can make the characteristics closer to those in the experiment. In addition, replacing the vacuum layer in front of the antenna by realistic plasma and calculating the full mode propagation in the plasma domain as in [11], can give corrections to the local RF fields. On the other hand, it can be inferred from Fig. 2 that effects of the wave modes which propagate along the field lines do not qualitatively change the agreement between the calculations (in particular TOPICA) and the $i_{\mathrm{R}}$ measurement, although the latter implies connections along the field lines to the opposite side of the antenna. Such modes as the slow wave and the sheath-plasma wave are not accounted in the presented calculations.

On the measurement side, firstly, uncertainty in phasing can likely affect the measurements made at the antenna limiters. Some details will be discussed in the next section. Secondly, non-linear effects leading to density redistribution in front of the powered antenna can play a role for the local RF antenna characteristics. Thirdly, modifications of the $k_{||}$-spectrum can lead to additional effects, in particular due to the recirculating power which can be released in the SOL by the means of the unabsorbed fraction close to $k_{\|}=0$ for more imbalanced voltage configurations. However the corresponding modification of the $k_{\| \mid}$-spectrum is not extreme. Figure 3 shows $k_{\|}$power spectrum calculated by HFSS for the balanced and for the most extreme imbalanced configurations from Fig. 2, with only a minor fraction of the power close to $k_{\|}=0$ being affected. The symmetry of the spectrum is affected rather significantly though.

The effect of the strap voltage variation on the ICRF-specific $\mathrm{W}$ influx $\Delta \Gamma_{\mathrm{W}}$ measured on the LOSs at $a 4$ from Fig. 1 is presented in Fig. 4, along with the vertical dashed lines for the standard operational point and for the observed minimum of the $\mathrm{W}$ influx (for the LOSs where the minimum exists). As discussed in [2], it is problematic to describe differences in the absolute values of $\mathrm{W}$ influx from LOS to LOS, therefore we concentrate on the relative changes of $\mathrm{W}$ influx during the strap voltage ratio variation for a given LOS.

The ICRF specific W influx at LOSs 1 and 2 covering the a4 upper right corner, which is not connected along the field lines to the opposite (left) side of the antenna, strongly correlates with the increase of the local RF voltage at the $R$-strap. The W influx on these LOSs experiences a minimum close to $V_{\mathrm{RF} R} / V_{\mathrm{RF}}=0.5$, similar to the minimum for $i_{\mathrm{L}}$ and $i_{\mathrm{R}}$ in Fig. 2 and the minimum expected from the EM calculations. According to the EM calculations, the dependency of the local RF field amplitudes vs. strap voltage ratio

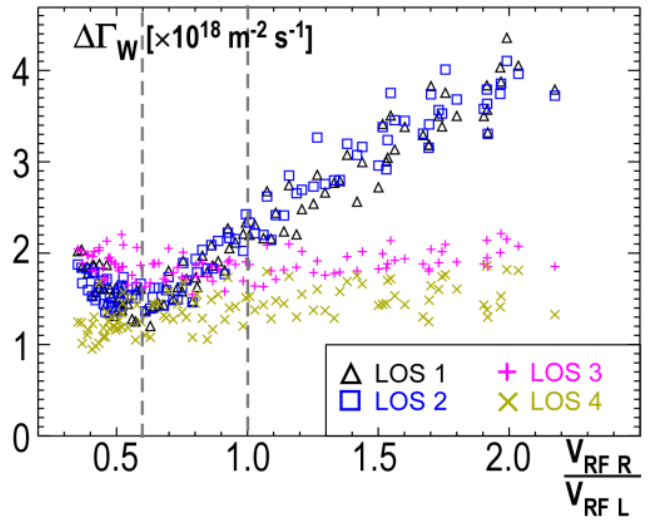

FIGURE 4. W influx vs. strap voltage ratio for a4. LOSs 1 and 2 are at upper right corner of $a 4$, LOSs 3 and 4 are close to equatorial plane of $a 4$ on right-hand side. AUG discharge \#31515. 
for the broad-limiter antenna $a 4$ (not shown) is similar to that for the original antenna $a 2$. The main differences between the antennas are: a) the RF field amplitude is generally lower at $a 4$; b) the variation of RF amplitudes during the voltage balance scan is smaller at $a 4$. The improvements of $a 4$ with respect to $a 2$ based on the calculations were reported in [2], along with the description of improvements observed in operation.

However one needs to stress that the $\Delta \Gamma_{\mathrm{W}}$ is not a direct measure of the RF fields at the antenna. A sputtering model for a known local light impurity charge state composition and an advanced RF sheath model are required to make a link between the RF fields and the sputtering characteristics. Nevertheless, the observed modulation of the $\mathrm{W}$ influx on LOSs 1 and 2 and the resemblance to the measurements of $i_{\mathrm{L}}$ and $i_{\mathrm{R}}$ at $a 2$ indicate that the local RF field amplitude plays an important role in $\mathrm{W}$ sputtering, at least for the given experimental conditions.

The situation is different for LOSs 3 and 4 which cover the locations connecting to the opposite side of the antenna along the magnetic field lines. Here, little variation of the $\mathrm{W}$ influx with the voltage strap ratio is observed. On one hand this would be consistent with the expectations from the EM calculations: closer to the antenna equatorial plane the local RF fields show less variation during the voltage balance scan. On the other hand, the opposite side of the antenna can impose plasma biasing to the field lines connected to the spots of observation for LOSs 3 and 4, resulting in stronger $\mathrm{W}$ sputtering. Thus the increase of the $\mathrm{W}$ influx at high local RF voltage could in principle be equalized by the plasma bias contribution of the opposite antenna side at low local RF voltage, so that little variation during the voltage balance scan is observed.

A strong plasma biasing by the antenna at the imbalanced configuration is implied from the measurements by the reciprocating RFA probe on the field lines connected to $a 4$. The measurements are presented in Fig. 5. Radial profiles of the averaged ion energies along magnetic field $<W_{\|, \mathrm{i}}>$ are measured in L-mode discharges at $B_{\mathrm{t}}=2.0 \mathrm{~T}$ with $P_{\mathrm{ICRF}}=0.5 \mathrm{MW}$ from two antennas at $30 \mathrm{MHz}$ during phases with various $\mathrm{q}_{95}$ values to resolve the poloidal dependence. The radial-poloidal profile is restricted to the lower half of $a 4$ due to geometrical boundary conditions of the probe placement. The parallel ion energies in the case of balanced strap voltages (upper graph of Fig. 5) are higher than $\sim 30 \mathrm{eV}$ measured in the whole region in ohmic phases, but do not exceed $80 \mathrm{eV}$ close to and in front of the antenna limiter where the particle flux is highest (see the density profile in Fig. 5, lower graph, as measured by Li-beam diagnostics). Only deep in the limiter shadow, where density is lower and error bars are higher, energies up to $130 \mathrm{eV}$ are observed. For the imbalanced voltage configuration (middle graph of Fig. 5), the measured energies are higher in the region with high particle flux. The energies exceed $140 \mathrm{eV}$ in the lower part of the antenna.

Assuming that the RFA measurements can be expressed in terms of the rectified DC potentials, the a4 antenna DC signatures corresponding to Fig. 5 can be modelled by the combination of TOPICA which produces RF field maps and the asymptotic SSWICH code which includes a model for slow wave propagation and an advanced sheath model [12]. For the AUG antenna boundary conditions, the qualitative features of the rectified potential profiles at the antenna can successfully reproduce the experimental features depending on the feeding scheme of the antenna [13]. These calculations confirm the additional plasma biasing in the imbalanced voltage

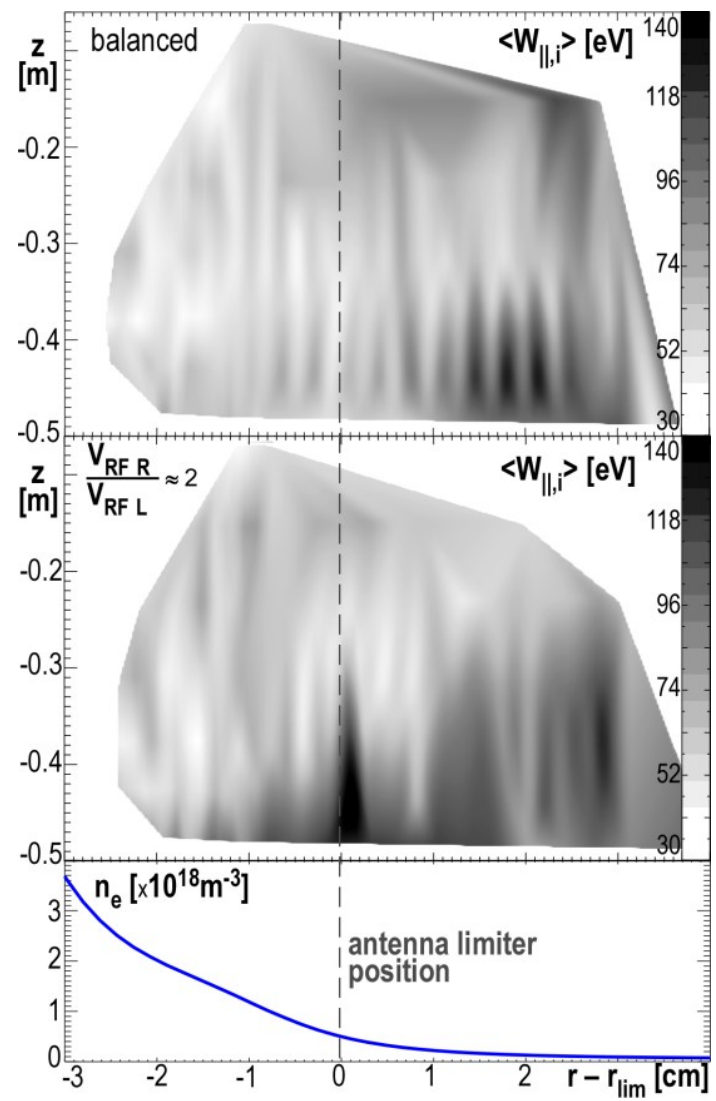

FIGURE 5. Ion energy measurements on field lines connected to $a 4$ for balanced (upper, AUG \#31278) and imbalanced configuration (middle, AUG \#31280) at dipole phasing. Lower graph: SOL density from Libeam diagnostics. configuration. Therefore it is possible that in the case of the experiments described above and corresponding to Fig. 2 and Fig. 4, the side of the antenna with low RF voltage and low local RF image current is affected by a plasma bias perturbation on the field lines connecting to the opposite antenna side. Thus the $\mathrm{W}$ source can also be affected, as discussed above. However other effects, described above in connection with the RF amplitude measurements, cannot be fully excluded. 
For a 2-strap antenna, the cancelation of the $(0, \pi)$-phased contributions of the RF image currents, considered responsible for the minima in the $i_{\mathrm{L}}$ and $i_{\mathrm{R}}$ measurements, cannot be achieved simultaneously on both sides of the antenna. The principle of the RF image current cancelation has driven the design of the new 3-strap antenna which will be tested in the 2015/2016 experimental campaign. For this antenna, it should be possible to reach the minimum of electric field on both left and right antenna sides simultaneously. The experiments will show if this is achievable for a wide range of parameters, in particular in the low density scenarios with more problematic $\mathrm{W}$ release.

\section{STRAP PHASING CHARACTERISTICS}

The strap feeder control system for $a 2$ and $a 4$ allows full $360^{\circ}$ phasing scan in a period of time as low as $2 \mathrm{~ms}$. Figure 6 presents a reaction of the ICRF specific $\mathrm{W}$ influx $\Delta \Gamma_{\mathrm{W}}$ at the LOSs described in Fig. 1 to a slow double $360^{\circ}$ scan of phasing $\Phi_{\mathrm{L}-\mathrm{R}}$ between $L$ and $R$ straps for the balanced voltage configuration, in the H-mode discharge with strong type-I ELMs at $B_{\mathrm{t}}=2.5 \mathrm{~T}, P_{\mathrm{NBI}}=5 \mathrm{MW}$, $P_{\mathrm{ECRH}}=1.3 \mathrm{MW}$ at $\mathrm{X} 2$ central heating and $P_{\mathrm{ICRF}} \approx 0.5 \mathrm{MW}$ at $36.5 \mathrm{MHz}$. The presence of strong central heating and limitation of ICRF power was found to be essential for the discharge to be radiatively stable for the phasing close to the monopole. Although in this experiment major deviations from the dipole $k_{\|}$spectrum are present, the reaction of $\Delta \Gamma_{\mathrm{W}}$ to the phasing scan follows the phasing almost linearly. The $\mathrm{W}$ influx on all LOSs from 1 to 4 is strongly modulated; modulation of LOS 3 and 4 is somewhat weaker. In addition to a better insight in the edge-RF interactions, this modulation during the phasing scan provides a useful experimental tool to control the $\mathrm{W}$

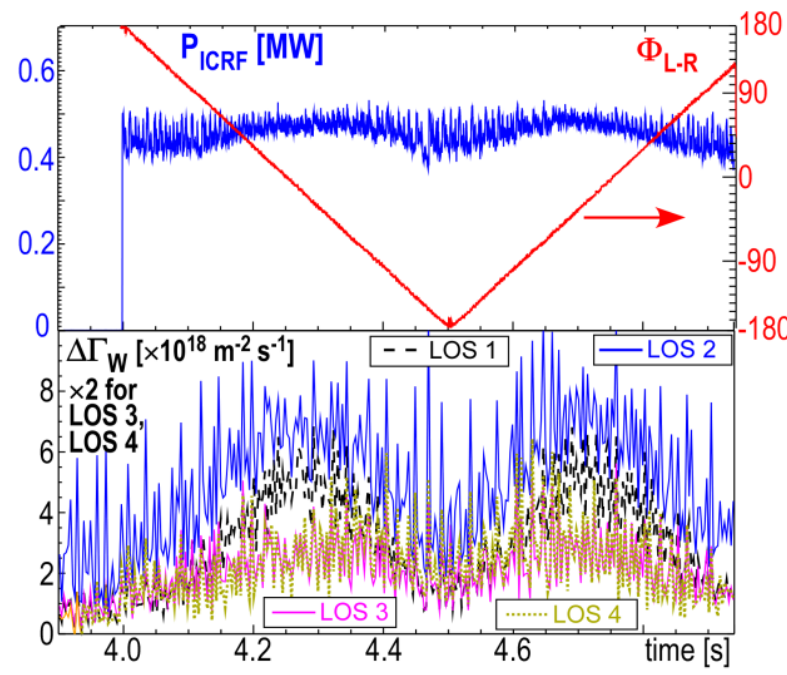

FIGURE 6. Time traces of reaction of the $\mathrm{W}$ source at $a 4$ to a double full $360^{\circ}$ phasing scan for AUG discharge \#30761. source which can be used in various other experiments, if $P_{\mathrm{ICRF}}$ is kept low with respect to other heating methods, in order to keep the effect of varying heating characteristics due to the $k_{\|}$spectrum variations at minimum.

The modulation of the ICRF specific $\mathrm{W}$ source during the $360^{\circ}$ phasing scans is qualitatively consistent with the fact that from the EM calculations the whole antenna is expected to be exposed to strong RF electric near-fields when the strap phasing is close to the monopole. To look closer on the applicability of the EM calculations to describe trends in the strap phasing characteristics, we adapt the same strategy as above: study the measurements of $i_{\mathrm{L}}$ and $i_{\mathrm{R}}$ in a discharge with suppressed ELMs. We also restrict the phasing scan to the range $+/-45^{\circ}$ around the dipole phasing, in order to limit the maximum $k_{\|}$spectrum modifications and have them similar to those in Fig. 3 (although less symmetric). The voltage balance ratio is kept close to 1 . The measurements and comparison with the EM calculations are presented in Fig. 7 versus deviation from dipole phasing $\Delta \Phi_{\mathrm{L}-\mathrm{R}}$. The measurements and the calculations are normalized at $\Delta \Phi_{\mathrm{L}-\mathrm{R}}=35^{\circ}$ for the best fit. Some features, like the presence of the minimum for $i_{\mathrm{L}}$ and the weaker variation of the amplitude for $i_{\mathrm{R}}$ than for $i_{\mathrm{L}}$, are similar both in the experiment and in the calculations, in particular in TOPICA. However the variation of RF amplitude in the experiment is considerably weaker than the variation in the EM calculations, especially for $i_{\mathrm{L}}$. This tendency can also be found for the strap voltage balance characteristics in Fig. 2 , but is much more visible for the phasing characteristics.

We hypothesize that one of the main candidates to explain the observed weak variation of the RF amplitude with phasing is an uncertainty
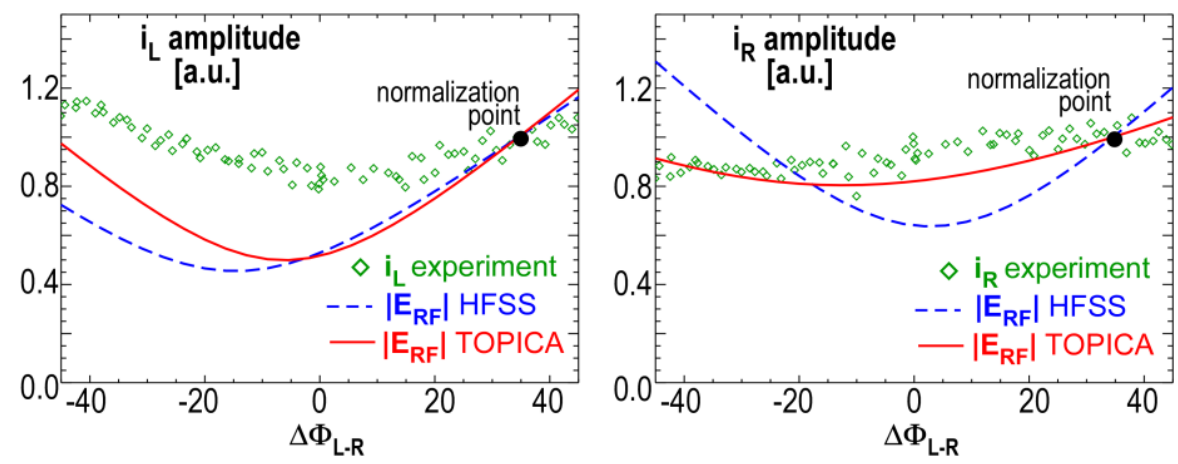

FIGURE 7. RF measurements $i_{L}$ (left) and $i_{R}$ (right) (AUG discharge \#31515) and field amplitudes from EM modeling of $a 2$ vs. deviation of strap phasing from dipole. 
of the phasing at the antenna plasma facing components. Firstly, systematic errors can be present: a) at $a 2$ due to the fact that the strap phasing is controlled based on the measurements in the transmission lines far from antenna; $b$ ) additional phase error at $a 4$ due to differences in the double-stub RF matching network and in the transmission line lengths. However these systematic errors in phasing would shift the minima expected from the calculations and would not reduce the sensitivity to the phasing scan. Secondly and more relevant, the phasing of the image currents at the antenna frame can be influenced by the plasma at the antenna plasma facing components. On one hand, this plasma can introduce local reactive elements such as sheath capacitance and plasma column inductance depending on the density fluctuations, and on the other hand, can lead to appearance of additional modes and mode mixing due to non-linearities. It can be shown that measurements of the phase difference between $i_{\mathrm{L}}$ and $i_{\mathrm{R}}$ have large fluctuations. This serves as an indication of the effect of the plasma on the phasing uncertainty.

Interestingly, it can be inferred from the EM calculations that the image current cancelation represented in the previous section by the minima in Fig. 2, is quite robust w.r.t. the phasing uncertainty. Even at large phasing deviations of $45^{\circ}$ the cancelation works, albeit not as efficiently. This can explain the better agreement between experiment and calculations for the strap voltage characteristics than that for the phasing characteristics. In fact, the lower sensitivity of the experimental curves in Fig. 2 compared to the curves from the calculations would be consistent with existence of a phasing uncertainty in the range of $10^{\circ}$ to $20^{\circ}$ degrees.

Certainly it is of advantage to minimize the phasing errors. To improve this, the new 3-strap antennas will have the phasing controlled based on the measurements inside the antenna. The antennas will also be equipped with a larger number of the RF measurements at the limiters to monitor the voltage balance and phasing of RF image currents thoroughly and optimize antenna settings.

\section{IMPROVEMENTS WITH LOCAL GAS INJECTION}

Independently of the antenna actuators such as strap voltage and phasing, the gas valve system in AUG provides the flexibility to affect the RF coupling and RF-related W release locally. In the experiments discussed below the gas valve system (see Fig. 1) was used to inject gas in one of the following configurations: from lower divertor in 4 locations; from top location in sector 2 or in sector 7; from the valve at the outer midplane in sector 3 or in sector 13. Apart from the case of the standard lower divertor valves with 4 injection locations used simultaneously, only one injection location at once was used here.

The relative improvement of antenna coupling resistance $\Delta R_{\mathrm{c}} / R_{\mathrm{c}}$ ref with respect to the case when the lower divertor valves are used, is shown in Fig. 8(left) for different gas valves as a function of the geometrical distance between antennas and valves. The use of the lower divertor valves has little influence on asymmetries in loading between the antennas and results in the lowest coupling resistance $R_{\mathrm{c} \text { ref. }}$. The discharges in question are type-I ELMy H-modes $B_{\mathrm{t}}=2.5 \mathrm{~T}$ with $P_{\mathrm{ICRF}}=1.5 \mathrm{MW}$ at $36.5 \mathrm{MHz}$ and $7.5 \mathrm{MW}$ total auxiliary power. $R_{\mathrm{c}}$ is estimated in the phases between ELMs, because these correspond to lowest and thus most critical values of $R_{\mathrm{c}}$. In all cases, a deuterium gas rate of about $1.2 \cdot 10^{22} \mathrm{el} / \mathrm{s}$ was used. The neutral pressure measured far away from the valves was monitored to be approximately the same for each valve.

The changes in the coupling resistance can be attributed to the density modifications in front of antenna, in the range close to the cut-off density. The strongest improvement of the antenna coupling resistance is observed when a
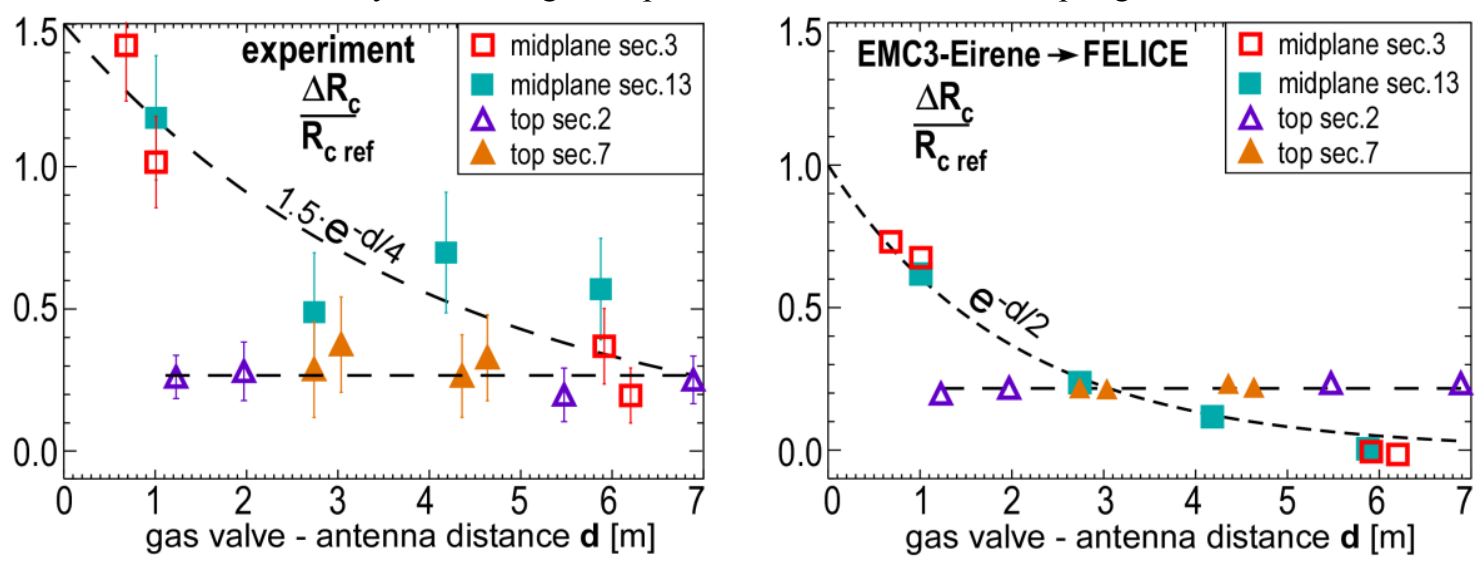

FIGURE 8. Left: Relative improvement of antenna resistance $\Delta R_{\mathrm{c}} / R_{\mathrm{c} \text { ref }}$ w.r.t. that in the case of lower divertor valves $R_{\mathrm{c} \text { ref }}$ vs. valve-antenna distance, with standard deviations indicated, from AUG discharges \#30634-30636, \#31269, \#31273. Right: Calculations of $\Delta R_{\mathrm{c}} / R_{\mathrm{c}}$ ref vs. valve-antenna distance based on the EMC3-Eirene - FELICE code combination. 
midplane valve is used close to an antenna. In these cases, improvement of the loading by $100 \%$ and even higher is possible. The $\Delta R_{\mathrm{c}} / R_{\mathrm{c}}$ ref dependency with the midplane valves tends to decrease exponentially with an e-folding length of about 4 meters, when the distance between the valve and antenna is increased. The top valves also improve coupling by $20-30 \%$, but little dependency on the geometrical distance between valve and antenna is seen. It was shown in [6] that the moderate and homogenous effect of the top valves on $R_{\mathrm{c}}$ is likely due to the fact that the magnetic field line connections from the region of the valves (slightly on the high field side) to the outer midplane are spread onto a large toroidal-polodial area.

Recently it became possible to model the effect of the density tailoring by local gas injection by the 3D SOL code EMC3-Eirene [7]. The details of the code setup and detailed description of the results can be found in [8]. The case of the plasma with the lower divertor gas valves is used as a reference. Edge plasma kinetic profiles of the reference experimental case are matched by those from the calculations by adjusting the local transport coefficients. Gas from the various valves is injected to study effect on the density tailoring. The calculations confirm that there are two major effects which can lead to a significant increase of electron density in front of the antennas: a) magnetic connection to a plasma density cloud in front of a gas valve; b) toroidal and poloidal spread of neutral density prior to ionization and subsequent ionization of the neutrals directly in front of the antenna. The latter is more pronounced for the valves at the outer midplane which are further away from the plasma in the radial direction. For the calculations presented below, the midplane valves were retracted significantly to the radial position close to the outer grid boundary. This corresponds to the valve placement in the AUG vessel.

In order to apply the calculations to the RF coupling studies, the density and temperature profiles from the EMC3-Eirene calculations are averaged in front of each antenna. The resulting edge profiles are combined with the measured core profiles to provide input for the 1D RF coupling code FELICE [14] which calculates the antenna resistance. The corresponding $\Delta R_{\mathrm{c}} / R_{\mathrm{c}}$ ref values, for the first time calculated with the help of the combination of EMC3-Eirene and FELICE, are presented in Fig. 8 (right) for the same cases as in the experiment.

The tendencies in the experiment are well-described by the calculations. Whereas the effect of the top valves on $\Delta R_{\mathrm{c}} / R_{\mathrm{c} \text { ref }}$ is almost independent from the valve position with $\Delta R_{\mathrm{c}} / R_{\mathrm{c}}$ ref lying between 0.2 and 0.3 , the effect of the midplane valves shows exponential decay with the valve-antenna distance. For the calculations, the e-folding length of the midplane valve dependence is about 2 meters. The $\Delta R_{\mathrm{c}} / R_{\mathrm{c}}$ ref values for the midplane valves from the calculations are generally below those in the experiment by an offset of $\sim 0.4$. The offset is likely the reason for the difference in the e-folding length. It is not understood yet what is responsible for this offset.

Future experiments plan to use L-mode discharges in order to provide the best SOL profiles possible for the calculations. Some aspects of the calculations can also be improved: a) detailing the structure of the components at the low field side of the grid of EMC3-Eirene in order to allow for the neutrals to penetrate toroidally and poloidally behind the structures; b) matching neutral pressure in the calculations to that measured in the experiments; c) using TOPICA for the coupling calculations instead of FELICE; d) assessing the influence of averaging procedure of 3D profiles to $1 \mathrm{D}$.

The local deuterium density tailoring affects beneficially the local $\mathrm{W}$ sources and $\mathrm{W}$ content in the plasma during the experiments. Local increase of the deuterium density reduces the $\mathrm{W}$ sputtering and $\mathrm{W}$ source, at least during ICRF operation. Figure 9 shows how the total time-averaged $\mathrm{W}$ influx (including ICRF related) measured at all LOSs from Fig. 1 reacts to switching between the valves for the conditions described above. When the midplane valve in sector 13, i.e. close to the observation location at $a 4$ is used, the $\mathrm{W}$ source is reduced significantly compared to the cases when either the remote midplane valve or any other type of valve is used. The global influence of the

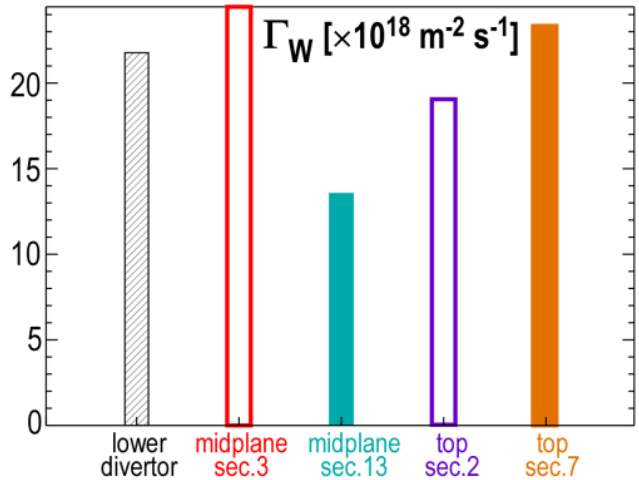

FIGURE 9. Reaction of the time-averaged $\mathrm{W}$ influx to switching between various valves. Data from AUG discharges \#30634-30636, \#31269, \#31273.

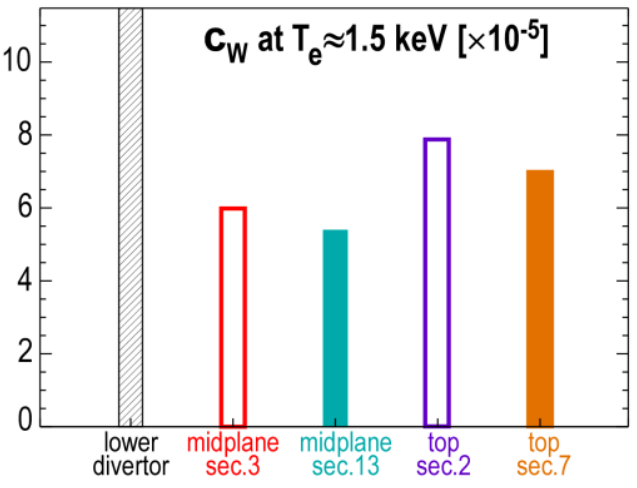

FIGURE 10. Effect of gas location on core W content. Data from AUG discharges \#30634-30636, \#31269, \#31273. 
gas injection location can be assessed from Fig. 10 which shows $\mathrm{W}$ concentration in the confined plasma in the region of $T_{\mathrm{e}} \approx 1.5 \mathrm{keV}$. Midplane valves are most efficient in increasing the density at the outer wall where the dominant $\mathrm{W}$ source is present. The largest $\mathrm{W}$ source is observed with the standard lower divertor valves which are less efficient in increasing the density close to the locations at the low field side.

The midplane valves are thus the valves of the first choice during ICRF operation, since they lead to improved coupling and reduced impurity release. However not every design of the midplane valve is suitable. As shown in [15], valves with the orifices close to the antenna mouth can lead to enhancement of non-linear effects and extremely local density increase. It is therefore preferable to place the orifices with a significant radial retraction, so that neutral can spread poloidally and toroidally before getting ionized.

\section{CONCLUSIONS}

Compensation of RF image currents expected from HFSS and TOPICA calculations, is evidenced by the RF measurements at the limiters of the 2-strap AUG ICRF antenna phased close to the dipole. The basic principle behind the design of the planned new 3-strap antenna is therefore confirmed experimentally. It is still to be tested whether the RF image currents can efficiently be compensated at a large plasma facing area of the new antenna simultaneously, so that the $\mathrm{W}$ source can be reduced significantly. Meanwhile for the 2-strap antenna, the W source measured at the locations not connected to the opposite antenna side along magnetic field lines, strongly correlate with the RF voltage at the local strap. The observations of RF amplitudes and $\mathrm{W}$ source are consistent with previous observations of heat flux at Tore Supra [1] and recent developments in theory [13, 16] stating that the local RF amplitude of parallel electric field plays the major role in causing the non-linear RF-plasma interactions, and not the field integrated along a magnetic field line. However the effect of the local RF field on the interactions is difficult to differentiate from the plasma biasing of the magnetic field lines connected to regions with high RF fields. Such plasma biasing is observed in AUG with a retarding field analyzer.

For the first time the effect of local gas injection on RF coupling and local $\mathrm{W}$ sources by means of deuterium density tailoring can be assessed theoretically with the help of SOL code EMC3-Eirene and coupling calculations by FELICE. A good agreement between the experiment and the calculations is found, except that estimates of the coupling resistance by FELICE using 1D plasma profiles averaged from the 3D profiles of EMC3-Eirene show lower values for the valves at the outer midplane compared to the experiment. Nevertheless the estimates confirm these valves to be most efficient in tailoring the density in front of the antennas.

\section{ACKNOWLEDGMENTS}

This work has been carried out within the framework of the EUROfusion Consortium and has received funding from the Euratom research and training programme 2014-2018 under grant agreement No 633053. The views and opinions expressed herein do not necessarily reflect those of the European Commission.

\section{REFERENCES}

1. $\quad$ L. Colas et al., J. Nucl. Mater. 438 (2013), S330-S333.

2. $\quad$ V. Bobkov et al., Nucl. Fusion 53 (2013), 093018.

3. S. Wukitch et al., Phys. Plasmas 20 (2013), 056117.

4. M.L. Mayoral et al., AIP Conf. Proc. 933 (2007), 55.

5. P. Jacquet et al., Nucl. Fusion 52 (2012), 042002.

6. P. Jacquet et al., Proc. 25th Int. Conf. on Fusion Energy (St. Petersburg, Russia, 2014), EX/P5-39.

7. Y. Feng, et al., Contrib. Plasma Phys. 44 (2004) No. 1-3, 57-69.

8. W. Zhang et al., this conference.

9. High Frequency Structure Simulator, www.ansys.com.

10. V. Lancellotti et al., Nucl. Fusion 46 (2006) S476.

11. J. Jacquot et al., this conference.

12. J. Jacquot et al., Phys. Plasmas 21,061509 (2014)

13. A. A. Křrivská et al., this conference.

14. M. Brambilla, Nuclear Fusion 35 (1995), 1265-1280.

15. V. Bobkov, et al., AIP Conf. Proc. 1580 (2014), 271.

16. L. Colas et al., this conference. 